\title{
Auditory distraction and acclimatization to hearing aids
}

DOI:

10.1097/AUD.0000000000000366

\section{Document Version}

Accepted author manuscript

Link to publication record in Manchester Research Explorer

\section{Citation for published version (APA):}

Dawes, P., \& Munro, K. (2016). Auditory distraction and acclimatization to hearing aids. Ear and hearing. https://doi.org/10.1097/AUD.0000000000000366

\section{Published in:}

Ear and hearing

\section{Citing this paper}

Please note that where the full-text provided on Manchester Research Explorer is the Author Accepted Manuscript or Proof version this may differ from the final Published version. If citing, it is advised that you check and use the publisher's definitive version.

\section{General rights}

Copyright and moral rights for the publications made accessible in the Research Explorer are retained by the authors and/or other copyright owners and it is a condition of accessing publications that users recognise and abide by the legal requirements associated with these rights.

\section{Takedown policy}

If you believe that this document breaches copyright please refer to the University of Manchester's Takedown Procedures [http://man.ac.uk/04Y6Bo] or contact uml.scholarlycommunications@manchester.ac.uk providing relevant details, so we can investigate your claim.

\section{OPEN ACCESS}




\section{Auditory distraction and acclimatization to hearing aids}

Objective: It is widely recognized by hearing aid users and audiologists that a period of auditory acclimatization and adjustment is needed for new users to become accustomed to their devices. The aim of the present study was to test the idea that auditory acclimatization and adjustment to hearing aids involves a process of learning to 'tune out' newly audible but undesirable sounds, which are described by new hearing aid users as annoying and distracting. It was hypothesized that: i) speech recognition thresholds in noise would improve over time for new hearing aid users; ii) distractibility to noise would reduce over time for new hearing aid users; iii) there would be a correlation between improved speech recognition in noise and reduced distractibility to background sounds; iv) improvements in speech recognition and distraction would be accompanied by self-report of reduced annoyance; $v$ ) improvements in speech recognition and distraction would be associated with higher general cognitive ability and more hearing aid use.

Design: New adult hearing aid users $(n=35)$ completed a test of aided speech recognition in noise (SIN) and a test of auditory distraction by background sound amplified by hearing aids on the day of fitting and 1, 7, 14 and 30 days post-fitting. At day 30, participants completed self-ratings of the annoyance of amplified sounds. Daily hearing aid use was measured via hearing aid data logging and cognitive ability was measured with the WASI block design test. A control group of experienced hearing aid users $(n=20)$ completed the tests over a similar time frame.

Results: At Day 30, there was no statistically significant improvement in SIN among new users versus experienced users. However, levels of hearing loss and hearing aid use varied widely among new users. A subset of new users with moderate hearing loss who wore their hearing aids at least 6 hours/day $(n=10)$, had significantly improved SIN (by $\sim 3 \mathrm{~dB}$ signal to noise ratio), compared to a control group of experienced hearing aid users. Improvements in SIN were associated with more consistent HA use and more severe hearing loss. No improvements in the test of auditory distraction by background sound were observed. Improvements in SIN were associated with self-report of background sound being less distracting and greater self-reported hearing aid benefit. There was no association between improvements in SIN and cognitive ability or between SIN and auditory distraction. 
33 Conclusions: Improvements in SIN were accompanied by self-report of background 34 sounds being less intrusive, consistent with auditory acclimatization involving a 35 process of learning to 'tune out' newly audible unwanted sounds. More severe 36 hearing loss may afford the room for improvement required to show better SIN

37 performance with time. Consistent hearing aid use may facilitate acclimatization to 38 hearing aids and better SIN performance. 
Auditory acclimatization is considered to be a process of perceptual learning, whereby an individual learns over time to make use of the change in acoustic information provided by the hearing aid (Arlinger et al. 1996). Clinicians and hearing aid users recognise a need for new hearing aid users to adjust to hearing aid use (Dawes, Maslin, et al. 2014; Mueller et al. 2001). Clinicians recommend that consistent hearing aid use is likely to lead to greater hearing aid benefit and satisfaction (Mueller and Powers 2001), and auditory acclimatization periods are commonly included in research studies. But there is inconsistent research evidence for auditory acclimatization with hearing aids. In relation to improved speech recognition, several studies reported evidence for acclimatization (Cox et al. 1992; Cox et al. 1996; Gatehouse 1992, 1993; Horwitz et al. 1997; Kuk et al. 2003; Munro et al. 2003; Yund et al. 2006), while others have not (Bentler et al. 1993a, 1993b; Dawes, Munro, et al. 2014a; Humes et al. 2003; Humes et al. 2002; Saunders et al. 1997; Taylor 1993). See Turner et al. (1996), Palmer et al. (1998), Munro (2008) and Dawes et al (2014a, 2014b) for reviews of these and other studies. The inconsistencies between previous studies may have been due to design factors, such as lack of control group, unilateral versus bilateral fitting, the type and amount of auditory input, inclusion of participants with mild hearing loss in some studies, the amount of hearing aid use and cognitive factors (Munro 2008; Palmer et al. 1998). Therefore, we previously conducted a large longitudinal study of auditory acclimatization to hearing aids that included a control group of experienced hearing aid users (with at least moderate levels of hearing loss), unilateral versus bilateral fittings and accounted for the amount of hearing aid use and cognitive factors (Dawes et al. 2013a, 2013b; Dawes, Munro, et al. 2014a, 2014b). New hearing aid users reported significant increases in benefit, but there was little corresponding evidence for perceptual or physiological changes with hearing aid use. These null findings lend weight to the views of some researchers who have concluded that because auditory acclimatization effects tend to be inconsistent between studies and small on average, they are clinically insignificant (Turner et al. 1998). However, this conclusion seemed at odds with the experience of clinical audiologists and hearing aid users. A possible explanation for the apparent discrepancy between research 
71 findings and the experience of clinicians and new hearing aid users may be that the current conceptualization of auditory acclimatization is inaccurate or incomplete.

In a focus group study, new hearing aid users were asked to describe the process of 'getting used to' hearing aids (Dawes, Maslin, et al. 2014). The primary obstacle reported by all users was that of becoming accustomed to a 'cacophony of sound', much of which had previously been inaudible for many years. Users reported that the experience of amplified background sound was initially overwhelming, but that after a few days of hearing aid use, these undesirable sounds ceased to be noticeable. This experience has been recognized previously in terms of 'annoying or aversive' sounds following amplification (Brooks 1989; Palmer et al. 2006; Skagerstrand et al. 2014). In a recent scoping review of reasons for non-use of hearing aids, five out of the ten studies that were identified for the review cited 'noisy situations/background noise' as a primary reason for non-use (McCormack et al. 2013), and background sound appears to be relevant to various aspects of hearing aid use, including benefit and satisfaction, acclimatization, hearing aid technology and signal processing (Gygi et al. 2015).

We hypothesize that auditory acclimatization may primarily involve a re-focus of auditory attention, as follows. Among people with normal hearing, audible background sounds with no behavioural relevance (such as ventilation noise, street noise, rustling clothes etc.) are ignored. In the case of people with long-standing hearing loss, background sounds are inaudible or very quiet. But when someone with long standing hearing loss obtains a hearing aid, the audibility of background sounds is suddenly restored. Immediately following hearing aid fitting, background sounds intrude on conscious attention due to their novelty. Newly audible background noises may exert a detrimental distracting effect on speech recognition (i.e. 'informational masking'). 'Informational masking' refers to degradation of auditory detection or discrimination of a signal that is not related to energetic masking caused by physical interaction between the masker and the signal (Leek et al. 1991). Informational masking is greater with more complex maskers and with maskers that are similar to the signal. Once someone with long standing hearing loss has acclimatized to hearing aids, irrelevant background sounds are ignored and informational masking is reduced. This concept is consistent with a description of auditory acclimatization as a process of perceptual learning (Arlinger et al. 1996). 
104 Alterations of attentional focus have been identified previously in research in auditory learning (Amitay et al. 2006). Amitay (2009) proposed a theory of auditory perceptual learning that includes attentional filtering of irrelevant stimulus features. This theoretical model may be applicable in the case of auditory acclimatization to hearing aids. Alteration in the focus of auditory attention may also be consistent with earlier reports that showed improvements in aided speech recognition performance over time (for example, Horowitz \& Turner, 1997; Munro \& Lutman, 2003; Yund et al., 2006). These previous studies measured speech recognition in background noise in order to avoid ceiling effects. Improvements in speech recognition in noise in these previous studies may have been due to reduced susceptibility to the distracting effects of noise rather than improved speech recognition per se. Other areas of research suggest that the distracting effect of background noise ('informational masking') has a significant impact on speech recognition, and that attention plays a key role in resistance to distraction effects (for example, Helfer \& Freyman, 2008). Other components that may influence post-hearing aid fitting improvement in speech recognition in noise may include modulation masking (Bacon et al. 1998), physiological changes (Dawes et al. 2013a; Dawes, Munro, et al. 2014b), 'listening effort' or top down cognitively-based compensatory function (Dawes et al. 2010; Pichora-Fuller et al. 2006; Wong et al. 2009) and reallocation of executive function capacity (Amitay 2009).

In the present study, we sought to index the distracting effect of background noise during aided listening using a task derived from cognitive psychology. A body of previous cognitive psychological research has shown that background noise impairs performance on cognitively challenging tasks, with memory-related tasks being particularly vulnerable (Cassidy et al. 2007; Jones et al. 2010). Participants therefore completed a serial recall memory task in quiet and in noise (with hearing aids), with the difference in recall between quiet and noise conditions being an index of the distracting effect of amplified background noise (the 'distraction' task).

If the importance of attentional tuning for hearing aid auditory acclimatization can be established, this could have relevance for both research and clinical practice. Failure to 'tune out' aversive sounds may be a primary reason for lack of auditory acclimatization, reduced hearing aid benefit and non-use. A better understanding of auditory acclimatization as a process involving re-focus of auditory attention may 
137 ultimately help hearing impaired people obtain more benefit from amplification.

138 Specifically, reliable measurement of altered focus of auditory attention with hearing aid use may be used to i) identify optimal hearing aid settings for first fits (e.g. optimisation of hearing aid 'acclimatization' first fit settings which begin with low levels of gain and gradually increase gain to prescribed levels over an extended time period) ii) inform strategies to facilitate auditory acclimatization (e.g. counselling and listening tasks, such as those described by Brooks, (1989)) iii) prescribe individualized management and fittings to optimize hearing aid benefit (e.g. more aggressive acclimatization settings for those who are particularly susceptible to the distracting effects of background noise).

In a study with older adults with hearing loss, Nabelek et al (1991) reported that full time hearing aid users tolerated higher levels of background noise than occasional or non-hearing aid users. Following Nabelek et al's (1991) study, Rogers et al (2003) described the Acceptable Noise Level (ANL) test, which requires test takers to listen unaided to spoken discourse presented at a comfortable listening level and adjust the level of background noise to the maximum level where they feel they can still follow the discourse. Nabelek et al. (2006) suggested that different acceptance of noise (indexed by the ANL test) may result in different patterns of hearing aid use. People who tolerate higher levels of background noise may use hearing aids more consistently. A recent review (Olsen et al. 2014) reported inconsistent evidence that the ANL was predictive of hearing aid use. Olsen et al (2014) concluded that acceptance of noise may be an important determinant of hearing aid use, but that the reliability of the ANL was not satisfactory. We hypothesized that 'tolerance of background noise' indexed by the ANL may relate to susceptibility to the distracting effect of background noise, as described in our conception of acclimatization to hearing aids above. We expected that higher susceptibility to background noise on the distractibility task measured at baseline would be associated with lower tolerance of background noise on the ANL test.

Study design and hypotheses

166 In the present study, new adult hearing aid users completed an experimental test 167 battery on the day of first hearing aid fitting and at 1, 7 and 30 days post fitting. On 168 the basis of reports by new hearing aid users (Dawes, Maslin, et al. 2014) it was 
anticipated that a re-focus of auditory attention with new hearing aid use may occur relatively rapidly, within the first few days of hearing aid use. Participants were tested at 1 and 7 days after fitting to capture this process. A final test session at 30 days was included to test for longer-term stability of any early changes and whether improvements may still be evident at 30 days post-fitting. In order to control for retest effects, testing included a control group of experienced adult hearing aid users. Most previous studies on acclimatization to hearing aids assessed aided speech recognition at fixed SNRs in order to avoid celling effects. In the present study, speech recognition was assessed with the level of background noise varying adaptively to track a recognition threshold. If auditory acclimatization to hearing aids results in an improved ability to ignore background sounds, we hypothesized that i) speech recognition thresholds would improve over time, with new hearing aid users achieving equivalent levels of recognition at more adverse SNRs over time post hearing aid fitting (hypothesis \#1) and ii) that new hearing aid users would show reduced distractibility over time (hypothesis \#2). If reduced distractibility to background sounds underlies improvements in speech recognition in noise, then reductions in distractibility should correlate with improvements in speech recognition (hypothesis \#3). Improvements in speech recognition and reduced distraction should also be accompanied by self-report of reduced annoyance (hypothesis \#4).

Finally, in terms of explaining individual differences in performance on a large range of laboratory tasks, most variance can be explained by general cognitive ability (Rabbit 2002). Individual differences in a variety of mental tests including executive function, attention, working memory and vocabulary are predominantly accounted for by general cognitive ability (Deary et al. 2010). Cognitive ability has also been suggested as a constraining factor in relation to auditory acclimatization (Palmer et al. 1998; Tyler et al. 1996) and for speech recognition in background noise (Akeroyd 2008). It is reasonable then to presume that general cognitive ability would be positively correlated with auditory attention and successful auditory acclimatization. As general cognitive ability may be estimated quickly, it may be a potentially useful clinical metric for individualization of hearing aid fitting. Further, if consistent hearing aid use facilitates learning to ignore background sound, more consistent hearing aid use would be associated with improvements in speech recognition and distraction. We hypothesized that improvements in speech recognition and distraction would be 
associated with higher general cognitive ability and more consistent hearing aid use (hypothesis \#5).

\section{MATERIALS AND METHODS}

\section{Participants}

Power analyses concluded that at least 17 new hearing aid users and 17 controls would provide at least $80 \%$ power to detect a large sized effect $(d=0.5$; based on a repeated measures ANOVA with an alpha level of 0.05 , two-tailed). A minimum sample size of 29 new hearing aid users would be required to detect a large correlation $(r>0.5)$ between improvement in distraction/speech recognition performance and hearing aid use/cognitive ability (based on Pearson's $r$ with an alpha level of 0.05). A total of 22 experienced and 42 new hearing aid users were recruited. 9 participants were excluded due to non-completion, change in hearing sensitivity or change in hearing aid gain. Participants who were excluded from the study did not differ from those who completed the study in terms of age $(\mathrm{t}(62)=0.2$, $p=0.80)$ or severity of hearing loss $(\mathrm{t}(62)=0.6, p=0.50) .20$ experienced and 35 new hearing aid users were included in the analysis. The mean age of the experienced and new user groups was 78 years (SD 8; 67-88 years) and 70 years (SD 10; 57-69 years), respectively. The mean hearing level (Figure 1; 250 to 8,000 $\mathrm{Hz}$ ) in the experienced and new user groups was $46 \mathrm{~dB} H L$ (SD = 7; range 21 to 55 $\mathrm{dB} H \mathrm{HL}$ ) and $38 \mathrm{~dB} H \mathrm{HL}$ ( $\mathrm{SD}=9$; range 31 to $59 \mathrm{~dB} \mathrm{HL}$ ). Experienced hearing aid users were older $(t(53)=2.98, p<0.01)$ and had poorer hearing than new hearing aid users $(\mathrm{t}(53)=3.28, p<0.01)$.

(Insert FIGURE 1 here)

Participants were recruited to the study, fitted and tested by an audiologist based at Withington Community Hospital, a local NHS audiology clinic. Ethics approval was obtained from the North West UK National Health Service (NHS) Research Ethics Committee. In the UK, hearing aids are currently provided free of charge at the point of service via the NHS. Recruitment aimed at including an inclusive range of new hearing aid users. Our recruitment targeted new hearing aid users who have elected to receive hearing aids as part of their standard clinical care. 
233 We also recruited experienced hearing aid users with at least 12 months experience

234

235

236

237

238

239 and who report hearing aid use $\geq 6$ hours per day. Inclusion criteria for all participants was mild-moderate hearing loss consistent with normal aging (defined as average air conduction thresholds across 500,1000, 2000 and $4000 \mathrm{~Hz}>20 \mathrm{~dB} \mathrm{HL}$ and $<70 \mathrm{~dB}$ $\mathrm{HL}$ ). Exclusion criteria were i) fluctuating or recent changes in hearing level, ii) asymmetry in air conduction thresholds of greater than $15 \mathrm{~dB}$ at two or more frequencies, iii) an air-bone gap greater than $15 \mathrm{~dB}$ at any test frequency and iv) abnormal middle ear function assessed using tympanometry. Hearing thresholds were assessed at the start and end of the study, and participants with any substantial change in threshold ( $>10 \mathrm{~dB}$ over more than one test frequency) excluded from analysis $(n=2)$. Mean change in threshold across frequencies and groups was $0.5 \mathrm{~dB}(\mathrm{SD}=1.0)$. Overall, $2 \%$ of thresholds changed by $10 \mathrm{~dB}$ or more. This is in line with test-retest differences for audiometry (Robinson 1991) and similar to our previous acclimatization studies with a similar patient population (Dawes, Munro, et al. 2014a).

\section{Hearing aid fitting}

New hearing aid users were all bilaterally fitted with the same model of hearing aid that is routinely used for cases of mild-moderate hearing loss at Withington Hospital's Audiology department (Oticon Spirit Zest; a digital nonlinear behind-the-ear open fit hearing aid). The hearing aids have 16 channel signal processing with a frequency range of $100-7100 \mathrm{~Hz}$. Noise management was activated. Compression operated with an attack time of $2 \mathrm{~ms}$ and release time of 70 ms (per American National Standards Institute standard (ANSI S3.22 2003)).

All new hearing aids were fitted to the National Acoustics Laboratories Nonlinear 2 (NAL-NL2) prescription target based on hearing thresholds as measured at day 0 . Existing hearing aid users had hearing aids which had been previously fitted to either the NAL-NL1 or NAL-NL2 prescription target. Maximum output was adjusted if the participant reported any undue discomfort to loud sounds presented during the hearing aid fitting. Real ear insertion gain (REIG) was measured for all participants at the start and end of the study with a Siemens Unity 2 hearing aid analyzer following recommended procedure (British Society of Audiology and the British Academy of Audiology 2008). Target and measured user gain for the experienced and new user 
265 group is shown in Figure 2. Measured REIG levels were higher for experienced compared to new users. These higher values could be attributed to a slight trend for more severe hearing loss in the experienced users. Participants did not have access to volume controls, and no alterations in gain were made during the study, and participants with measured alterations in gain $(>10 \mathrm{~dB}$ over more than 1 test frequency) between baseline and completion of the study were excluded from analysis $(n=4)$. Mean changes in REIG between baseline and completion of the study ranged from $-0.6 \mathrm{~dB}(\mathrm{SD}=2.9)$ to $1.1 \mathrm{~dB}(\mathrm{SD}=2.5)$ across frequencies; the mean change in REIG was not statistically significant based on repeated-measures ANOVA ( $p$ 's $<0.05)$. Variation in gain was consistent with short-term test-retest REIG data (Hawkins et al. 1991). For new hearing aid users, the average hours of use per day was recorded from hearing aid data logging at the end of the study. Data logging was not available for the experienced hearing aid users.

(FIGURE 2 here)

\section{Protocol}

New users were tested on the day of fitting and at 1, 7 and 30 days post fitting. The same order of testing was used at each test session. Experienced hearing-aid users were tested at the same time points to control for the possible effects of repeating the tests on performance. The test protocol is summarized in Table 1.

\section{(TABLE 1 here)}

\section{Outcome measures}

Speech in Noise (SIN) recognition

Institute of Electrical and Electronics Engineers (IEEE) sentences were presented at $65 \mathrm{~dB}$ SPL from a center speaker (at 0 degrees azimuth and ear level) with background noise at an initial SNR of $+4 \mathrm{~dB}$. The background noise was selected from a corpus of sound recordings of non-speech background noise from a variety of real-world settings from the Database of Annotated Real Environmental Sounds (DARES; van Grootel et al. 2009) in order to maximize ecological validity. Six different recordings of background noise from the DARES database were used 
296 including 'cafeteria noise', 'supermarket noise', 'courtyard noise', 'ventilation noise' and two different recordings of 'office noise'. The background noise type for each speech recognition task run was randomly selected. No recording contained any intelligible speech. Background noise was presented from two loud speakers placed symmetrically at \pm 45 degrees azimuth. The level of background noise was varied adaptively contingent on correct identification of all the content words in the target sentence, with the $50 \%$ correct recognition threshold being estimated. The initial step

Auditory distraction task reversals. The final eight reversals were averaged to provide the SNR for $50 \%$ criterion performance.

The basis of the auditory distraction task was a visual serial recall task, previously used in research on auditory distraction (Cassidy and MacDonald 2007). After a delay of 8 seconds, participants were required to recall a series of 8 digits presented in sequence on a visual display. Following a practice run, participants performed the recall task i) in background noise with hearing aids and ii) without hearing aids in quiet. Background noise was presented via two symmetrically located loud speakers (as for the SIN task; \pm 45 degrees azimuth) at $55 \mathrm{~dB}$ SPL. The $55 \mathrm{~dB}$ SPL presentation level was chosen to represent low level background noise comparable to noise levels typically experienced in home and department store settings (Pearsons et al. 1977). The background noise was the same six different noise recordings from the DARES database (van Grootel et al. 2009) as used with the speech in noise recognition test. A different noise was used for each run. A total of 12 trials in each condition (noise/quiet) were presented, 8 digits in each trial giving with a maximum score of 96 correct in each condition. Trials were presented in two blocks of 6 , with the order of presentation counter balanced across participants (i.e. noise/quiet/noise/quiet or quiet/noise/quiet/noise). The measure of distraction was calculated as digit recall performance in quiet minus performance in background noise (\% correct). After completing the distraction task, participants rated the annoyance and distraction of the background noises using a rating system by Palmer et al. (2006); How annoying did you find the background sounds played during the memory task? and How distracting did you find the background sounds played during the memory task? Participants rated annoyance and distraction on a 
32910 point Likert scale with descriptive anchors 'Not annoying at all'/'Not distracting at

330 all' at 0 to 'Very annoying'/'Very distracting' at 10.

331 Acceptable Noise Level (ANL)

332 The ANL (Fry Electronics Inc., Tigard OR) was administered without hearing aids as

333 per the instructions for the ANL. A target story was played at $55 \mathrm{~dB}$ SPL over a loud

334 speaker. The participant adjusted the level of the story in $2 \mathrm{~dB}$ steps until the level

335 was at the most comfortable listening level (MCL). The target story was then

336 presented at the participant's MCL, and the participant asked to adjust the level of

337 background noise (BNL) until the participant felt they could only just follow the target

338 story. The ANL was taken as MCL minus BNL. Smaller ANL values correspond to

339 greater tolerance of background noise.

340 Self-report measures

341 On completing the study, participants completed two self-report measures: 1 . The

342 International Outcome Inventory-Hearing Aids (IOI-HA) questionnaire, which

343 includes 7 questions relating to use, benefit and residual hearing difficulty (Cox et al.

344 2002); 2. A self-report questionnaire about changes in the 'annoyance' of amplified

345 sounds and awareness of the HA over the course of the study (Palmer et al., 2006).

346 Awareness of the hearing aid was defined as the proportion of the time the

347 participant reported being consciously aware of wearing the hearing aid. The

348 questions in the self-report questionnaire were as follows:

349 1. Compared to when you first started using your hearing aid, how noticeable do

350 you find background sounds (e.g. wind, rustling clothes or hair, footsteps,

$351 \quad$ mechanical or electrical sounds)?

352 2. Compared to when you first started using your hearing aid, how annoying do

353 you find background sounds in quiet environments, such as a quiet office or room?

3. Compared to when you first started using your hearing aid, what proportion of the time are you consciously aware that you are wearing it?

Responses were recorded on a Likert scale from -5 to +5 , with descriptive anchors

'Much more noticeable', 'Unchanged' and 'Much less noticeable' at $-5,0$ and +5 , respectively. Responses on these three questions were summed to create a variable named 'Self-reported change in aided sound'. 
362 Wechsler Abbreviated Scale of Intelligence-II (WASI-II)

New hearing aid users completed the block design subtest of the WASI-II (Wechsler

2011) as an index of general cognitive ability which has previously been used in hearing aid acclimatization studies (Dawes et al. 2013b; Dawes, Munro, et al. 2014a). The block design test involves timed construction of a geometric pattern using coloured blocks.

\section{RESULTS}

370

All data were checked to ensure normality of distribution and that data met the assumptions for parametric testing.

\section{Speech in noise recognition (SIN)}

There was a significant improvement over time for all participants (Figure 3a; main effect of time; $F(3,49)=3.20, p=0.02)$, but no significant time* group interaction $(F(3,49)=0.94, p=0.94)$. In other words, in the new user group as a whole, there was no statistically significant improvement in SIN compared to the control group. This pattern of results is consistent with a general practice effect. There was no evidence for an acclimatization effect which was hypothesized to have been observable in the form of greater improvement in the new user group. However, improvement in SIN among the new user group was associated with severity of hearing loss and with hearing aid use (see 'Correlates of improvements in speech in noise recognition' below). The new user group included participants with mild levels of hearing loss, and hearing aid use varied widely (0.3 to 14 hours per day). We therefore applied selection criteria for severity of hearing loss and hearing aid use previously used in auditory acclimatization studies (pure tone average $>40 \mathrm{~dB} \mathrm{HL}$ and daily hearing aid use $>6$ hours per day; criteria as recommended by Palmer et al 1998 and used by Dawes et al 2013a, 2013b and Dawes et al 2014a, 2014b) and reran the analysis for the participants that fit these selection criteria (new users $\mathrm{N}=10$; experienced users $\mathrm{N}=16$; Figure $3 \mathrm{~b}$ ). There was a significant improvement over time $(F(3,22)=7.21, p<0.01)$ and a significant time ${ }^{*}$ group interaction $(F(3,22)=4.55, p=$ 0.01). This pattern of results is consistent with an acclimatization effect in the form of greater improvement for new hearing aid users (with hearing loss $>40 \mathrm{~dB} \mathrm{HL}$ and who use their hearing aids $>6$ hours per day) compared to a control group of 
400

401

402

403

404

405

406

407

408

409

410

411

412 experienced hearing aid users. Comparing performance within the subset of new users for Day 1, Day 7 and Day 30 versus the day of fitting, there was a significant improvement at Day 30 only $(\mathrm{t}(25)=3.16, p<0.01)$. The size of improvement in the new user group was $4.1 \mathrm{~dB}$, versus $0.8 \mathrm{~dB}$ in the experienced user group. An improvement of $\sim 3 \mathrm{~dB}$ in SIN may therefore be attributable to acclimatization in this sub-group of new hearing aid users.

(FIGURE 3 here)

\section{Distraction task}

Distraction scores (Figure 4) were expected to progressively decrease over time (i.e. high positive scores at initial fitting and low positive scores at Day 30 . The pattern of performance actually showed a small positive score at Day 0 , with mixture of small positive and negative scores at different time points; negative scores indicate better performance in noise conditions versus quiet. The average size of the absolute difference between conditions was small (ranging between 0 to $8 \%$ across groups and across test occasions).

There was a statistically significant main effect of time $(F(3,49)=4.60, p<0.01)$, but no significant time* group interaction $(F(1,49)=1.89, p=0.14)$. This pattern of results is consistent with a general practice effect. There was no significant correlation between distraction and ANL $(r=-0.33, p=0.06)$. There were no significant correlations between distraction scores on consecutive test occasions ( $r$ 's 0.02-0.30, $p>0.05$; two-tailed). In contrast to SIN, there was no association between change in distraction between Day 0 and Day 30 and severity of hearing loss and hearing aid use or any other factor ( $r$ 's 0.07-0.32, p>0.05). There was no significant effect of time or time*group interaction for the subset of new hearing aid users with hearing loss $>40 \mathrm{~dB} \mathrm{HL}$ and who use their hearing aids $>6$ hours per day (time: $(\mathrm{F}(3,22)=2.48, p$ $=0.09)$, time* ${ }^{*}$ roup: $(\mathrm{F}(3,22)=1.46, p=0.25)$.

(FIGURE 4 here) 
424 Examining self-report of annoyance and distraction in relation to the distraction task, experienced users tended to rate the background noise as being more annoying and distracting than new users (Figure 5). There was no significant change in distraction or annoyance ratings between Day 0 and any subsequent time point for either new users or experienced users ( $p$ 's > 0.05; Wilcoxon signed rank test).

(FIGURE 5 here)

431

\section{Correlates of improvements in speech in noise recognition}

There was no association between improvement in SIN and change in distraction or WASI score among new hearing aid users $(n=35 ; r=-0.18, p=0.30 ; r=0.26, p=$ 0.13). Improvements in SIN were associated with poorer hearing, greater hearing aid use and self-report of background sounds being less noticeable or annoying (Figure 6). Improvements in SIN were positively associated with hearing aid benefit (IOI-HA). (FIGURE 6 here)

\section{DISCUSSION}

Hypothesis \#1. Speech recognition thresholds in noise would improve with time post hearing aid fitting for new hearing aid users

We observed an improvement in SIN for a subset of new hearing aid users (with pure tone average $>40 \mathrm{~dB} \mathrm{HL}$ and $\mathrm{HA}$ use $>6$ hours/day) versus experienced hearing aid users tested at 30 days after first hearing aid fitting. Greater improvement in SIN was associated with greater hearing aid benefit (IOI-HA). Following reports from hearing aid users (Dawes, Maslin, et al. 2014), we had expected that SIN improvements associated with reduced intrusiveness of 'annoying sounds' may occur within the first week of hearing aid use. However, the improvement observed in the present study for a subset of new hearing aid users (with pure tone average $>40 \mathrm{~dB} \mathrm{HL}$ and HA use $>6$ hours/day) emerged at the test point 30 days after fitting (Figure 3). Previous studies also suggested that improvements may not emerge until after at least several weeks after hearing aid 
454 fitting (Munro 2008). In the present study, aided SIN was not measured beyond 30 days post fitting. In retrospect, it would have been useful to include longer term measures to chart the time course of changes after 30 days post fitting.

457 In the analysis of a subset of new hearing aid users (with pure tone average $>40 \mathrm{~dB}$ $458 \mathrm{HL}$ and HA use $>6$ hours/day), mean performance is at a similar level to that of experienced users at Day 0; however, by Day 30, new users have better performance than experienced users. One might have expected new users to begin with poorer SIN, and improve to the level of experienced users. Possible reasons for experienced hearing aid users' poorer SIN performance are first, experienced hearing aid users were older and had poorer hearing thresholds than new users (cf Cox and Alexander, 1992). Both age and average hearing threshold were correlated with SIN performance in the study sample $(r=0.48, p<0.01 ; r=0.26 p<0.05$, respectively). Second, the consistency of hearing aid use in experienced users could not be confirmed with data logging; the experienced users may use their hearing aids less consistently than the subgroup of new users selected on the basis of HA use being $>6$ hours/day.

Previous research on auditory acclimatization to hearing aids has been inconsistent

471 (Turner and Bentler 1998). In our previous auditory acclimatization study, we did not detect any improvements in SIN in new hearing aid users compared to controls (Dawes, Munro, et al. 2014a). In the present study, we reported a large improvement $(\sim 3 \mathrm{~dB})$ in a subgroup of new hearing aid users (with pure tone average $>40 \mathrm{~dB} H \mathrm{HL}$ and HA use $>6$ hours/day) over controls. Possible reasons for the difference between the present study and our previous (2014a) auditory acclimatization study may relate to i) the use of a subgroup of new users in the present study who were consistent hearing aid users and had more severe levels of hearing loss, and/or ii) use of a different measure of speech recognition in noise. In the present study, naturalistic recordings of background noise were used rather than broadband noise typically used in previous studies. While it is possible that the use of different noise types on each run of the speech test may have affected the results, our baseline measures showed that there were no significant differences in speech-in-noise thresholds obtained with the different noise types. Hearing aid users may learn to ignore naturalistic background noise, but could be less likely to ignore broadband noise and similar noise maskers not usually encountered in daily life. Further, in the 
487 present study, the intensity of noise varied adaptively to track recognition threshold;

488 Dawes et al (2014a) and other previous studies (e.g. Munro \& Lutman 2003) assessed accuracy of speech recognition at fixed SNRs.

Hypothesis \#2. New hearing aid users would show reduced distractibility to noise over time and Hypothesis \#3. Improved speech recognition in noise would be associated with reduced distractibility to background sounds

New users did not show reduced distractibility on a digit recall task over time compared to the control group of experienced users. On Day 0, the distracting effect of background noise of digit recall was small; 3\% on average across participant groups. Because there was no strong distracting effect at the beginning of the study, the distraction task may not have been sensitive to small changes in the distracting effect of background noise.

There were also no changes on self-report of the annoyance or distraction ratings of the background noise used during the distraction task. Contrary to expectation, experienced users rated the background noise as being more annoying and distracting during the distraction task than new users did. As discussed above in relation to speech recognition in noise, the difference in annoyance and distraction ratings between experienced and new users may be due to experienced users being older and having poorer hearing than new users. Investigation revealed no association between age or hearing loss and annoyance or distraction ratings ( $r$ 's $<0.01$, ns), so it seems unlikely that the difference in ratings is due to group differences in age or hearing. A possible explanation for the difference between groups may be that new users' lower ratings of distraction and annoyance may be due to a 'halo effect'. The initial experience of increased audibility may lead to more favourable subjective ratings of aided listening by new hearing aid users than experienced hearing aid users who may experience ongoing hearing difficulties in background noise (Malinoff et al. 1989; Taylor 1993).

514 The distraction task, SIN and ANL all share the requirement to focus on a target while ignoring distracting background sound (i.e. focused auditory attention). One would therefore have expected performance on the tasks to be correlated. However, we found that the distraction task, SIN and ANL did not correlate with each other.

518 One explanation may be that they do not tap a common underlying factor (i.e. do not 
519 relate to auditory attention). However, our interpretation was that the ANL and the distraction test may not be reliable measures. One recent review paper identified large differences on re-test and low re-test reliability for the ANL (Olsen and Brännström 2014). For the distraction task, retest correlations were small and mostly non-significant (cf the SIN test with retest $r$ 's $>0.7$ ), the size of the effect of distraction was small on average $(<8 \%)$, and at some time points, the difference between performance in the two conditions was in the opposite direction to expected (i.e. better performance in noise than in quiet). The research audiologist who administered the distraction task reported that 'lapses' in performance were common. Participants would perform consistently, but then fail several trials in sequence after having lost concentration. Older people are known to have more variable task performance than younger people (MacDonald et al. 2009). Participants in the present study were aged around 70 years, and participants' variable performance (reflected by lapses of concentration) may have adversely affected the reliability of the distraction task. The task was based on a paradigm used in a large body of research into the distracting effects of background noise (Cassidy and MacDonald 2007; Jones et al. 2010). However, previous research with

536 this paradigm has mostly been carried out with undergraduate student participants.

537 For example, Cassidy \& McDonald (2007) reported a large effect of noise on a memory task, with worse immediate recall in noise versus quiet (effect size $r=0.7$ versus $r=0.05$ in the present study). Although the distraction task used in the present study was piloted with older adults, performance of this task by a clinical sample of older hearing impaired participants may be much more variable than for undergraduate participants. We therefore conclude that the distraction task was not a reliable measure in the present study. In the face of the apparent unreliability of the

544 distraction task, we must rely on self-report measures as an alternative index of

545 'distraction' (hypothesis \#3). It would be desirable to identify a reliable behavioural

546 measure of 'distraction' that would be applicable to older hearing impaired

547 participants and which could facilitate further investigation into acclimatization to

548 hearing aids based on the distracting effects of amplified background sound.

549 Hypothesis \#4. Improvements in speech recognition and distraction would be 550 accompanied by self-report of reduced annoyance 
551 Improvements in SIN were associated with self-report of reduced annoyance of

552 amplified background sound and reduced consciousness of wearing a hearing aid.

553 This was consistent with the hypothesis of reduced intrusiveness of background 554 sounds being linked to improvements in SIN performance. However, self-report 555 measures are susceptible to bias (Dawes et al. 2011) so due caution is warranted in 556 interpretation. We suspect that there was no association between self-report of 557 reduced annoyance and change in distraction on the behavioural task because of

558 the unreliability of the behavioural task (as described above).

Hypothesis \#5. Improvements in speech recognition and distraction would be associated with higher general cognitive ability and more consistent hearing aid use Improvements in SIN were not associated with higher general cognitive ability. More consistent hearing aid use was associated with improvement in speech recognition.

Severity of hearing loss was also a predictor of improvement in speech recognition, perhaps because potential gains in speech recognition are greater for those with more severe hearing loss. Consistent hearing aid use could support greater auditory acclimatization and benefit (Mueller and Powers 2001; Palmer et al. 1998).

\section{Conclusions}

Improvements in aided speech recognition in noise that were observed in new hearing aid users in the present study at 30 days post hearing aid fitting may be predicted by severity of hearing loss and consistent hearing aid use. Improvements were greatest for new hearing aid users with more severe hearing losses and those who used their hearing aids consistently. Compared to a control group of experienced users, there was a significant improvement in aided speech recognition in noise over time in a subgroup of new HA users with more severe hearing loss who

576 used hearing aids consistently. Improvement in aided SIN was associated with

577 increased self-reported HA benefit. Improvement in aided SIN was also associated

578 with self-reported reduction in the intrusiveness of background sound. There were no 579 associations between improvement in SIN and the ANL or the distraction measure.

580 The ANL and distraction measures did not correlate with each other. 


\section{Acknowledgements}

583 This study was funded by the Industry Research Consortium (IRC). Thank you to

584 Jennifer Tinker, the research audiologist who collected the data for this study and to

585 Dr Fred Marmel who piloted the measures.

586

587

588

\section{References}

Akeroyd, M. A. (2008). Are individual differences in speech reception related to individual differences in cognitive ability? A survey of twenty experimental studies with normal and hearing-impaired adults. International Journal of Audiology, 47, S53-S71.

Amitay, S. (2009). Forward and reverse heirarchies in auditory perceptual learning. Learning \& Perception, 1, 59-68.

Amitay, S., Irwin, A., Moore, D. R. (2006). Discrimination learning induced by training with identical stimuli. Nature Neuroscience, 9, 1447-1448.

ANSI S3.22. (2003). Specification of hearing aid characteristtics. New York.

Arlinger, S., Gatehouse, S., Bentler, R. A., et al. (1996). Report of the Eriksholm workshop on auditory deprivation and acclimatization. Ear \& Hearing, 17, 87S-90S.

Bacon, S. P., Opie, J. M., Montoya, D. Y. (1998). The effects of hearing loss and noise masking on the masking release for speech in temporally complex backgrounds. Journal of Speech, Language, and Hearing Research, 41, 549-563.

Bentler, R. A., Neibuhr, D. P., Getta, J. P. (1993a). Longitudinal study of hearing aid effectiveness. I Objective measures. Journal of Speech, Language and Hearing Research, 36, 808-819.

Bentler, R. A., Neibuhr, D. P., Getta, J. P. (1993b). Longitudinal study of hearing aid effectiveness. II Subjective Measures. Journal of Speech, Language and Hearing Research, 36, 820-831.

British Society of Audiology and the British Academy of Audiology. (2008). Guidance on the use of real ear measurement to verify the fitting of digital signal processing hearing aids.

Brooks, D. N. (1989). Adult aural rehabilitation. London: Chapman and Hall.

Cassidy, G., MacDonald, R. A. R. (2007). The effect of background music and background noise on the task performance of introverts and extraverts Psychology of Music, 35, 517-537.

Cox, R. M., Alexander, G. C. (1992). Maturation of hearing aid benefit: Objective and subjective measurements Ear \& Hearing, 13, 131-134.

Cox, R. M., Alexander, G. C. (2002). The International Outcome Inventory for Hearing Aids (IOI-HA): psychometric properties of the English version. International Journal of Audiology, 41, 30-35.

Cox, R. M., Alexander, G. C., Taylor, I. M., et al. (1996). Benefit acclimatization in elderly hearing aid users. Journal of the American Academy of Audiology, 7, 428-441.

Dawes, P., Maslin, M., Munro, K. (2014). Getting used to hearing aids from the perspective of adult hearing aid users. International Journal of Audiology, 53, 861-870.

Dawes, P., Munro, K., Kalluri, S., et al. (2013a). Brainstem processing following unilateral and bilateral hearing-aid amplification. NeuroReport, 24, 271-275.

Dawes, P., Munro, K., Kalluri, S., et al. (2013b). Unilateral and bilateral hearing aids, spatial release from masking and auditory acclimatization. Journal of the Acoustical Society of America, 134, 596-606.

Dawes, P., Munro, K., Kalluri, S., et al. (2014a). Acclimatization to hearing aids. Ear \& Hearing, 35, 203-212. 
Dawes, P., Munro, K., Kalluri, S., et al. (2014b). Hearing aid use-related auditory acclimatization: Late auditory evoked potentials and speech recognition following unilateral and bilateral hearingaid amplification Journal of the Acoustical Society of America, 135, 3560-3569.

Dawes, P., Munro, K. J., Kalluri, S., et al. (2010). Older adults, listening effort and hearing aids. In International Hearing Aid Conference, Lake Tahoe. Lake Tahoe.

Dawes, P., Powell, S., Munro, K. J. (2011). The placebo effect and the influence of participant expectation on hearing aid trials. Ear \& Hearing, 32, 767-774.

Deary, I. J., Penke, L., Johnson, W. (2010). The neuroscience of human intelligence differences. Nature Reviews Neuroscience, 11, 201-211.

Gatehouse, S. (1992). The time course and magnitude of perceptual acclimatization to frequency responses: Evidence from monaural fitting of hearing aids. Journal of the Acoustical Society of America, 92, 1258-1268.

Gatehouse, S. (1993). Role of perceptual acclimatization in the selection of frequency responses for hearing aids. Journal of the American Academy of Audiology, 4, 296-306.

Gygi, B., Hall, D. A. (2015). Background sounds and hearing-aid users: A scoping review. International Journal of Audiology.

Hawkins, D., Alvarez, E., Houlihan, J. (1991). Reliability of three types of probe tube measurements. Hearing Instruments, 42, 14-16.

Helfer, K. S., Freyman, R. L. (2008). Aging and speech-on-speech masking. Ear \& Hearing, 29, 87-98.

Horwitz, A. R., Turner, C. W. (1997). The time course of hearing aid benefit. Ear \& Hearing, 18, 1-11.

Humes, L. E., Wilson, D. L. (2003). An examination of changes in hearing-aid performance and benefit in the elderly over a 3-year period of hearing-aid use. Journal of Speech, Language and Hearing Research, 46, 137-145.

Humes, L. E., Wilson, D. L., Barlow, N. N. (2002). Changes in hearing-aid benefit following 1 or 2 years of hearing-aid use by older adults. Journal of Speech, Language and Hearing Research, 45, 772-782.

Jones, D. M., Hughes, R. W., Macken, W. J. (2010). Auditory distraction and serial memory: The avoidable and the ineluctable. Noise and Health, 12, 201-209.

Kuk, F. K., Potts, L., Valente, M., et al. (2003). Evidence of acclimatization in persons with severe-toprofound hearing loss. Journal of the American Academy of Audiology, 14, 84-99.

Leek, M. R., Brown, M. E., Dorman, M. F. (1991). Informational masking and auditory attention. Perception and Psychophysics, 50, 205-214.

MacDonald, S. W. S., Li, S. C., Bäckman, L. (2009). Neural underpinnings of within-person variability in cognitive functioning. Psychology and aging, 24, 792.

Malinoff, R. L., Weinstein, B. E. (1989). Changes in self-assessment of hearing handicap over the first year of hearing aid use by older adults. Journal of the Academy of Rehabilitative Audiology, 22, 54-60.

McCormack, A., Fortnum, H. (2013). Why do people fitted with hearing aids not wear them? International Journal of Audiology, 52, 360-368.

Mueller, H. G., Powers, T. A. (2001). Consideration of auditory acclimatization in the prescriptive fitting of hearing aids. Seminars in Hearing, 22, 103-124.

Munro, K. J. (2008). Reorganization of the adult auditory system: Perceptual and physiological evidence from monaural fitting of hearing aids. Trends in Amplification, 12, 254-271.

Munro, K. J., Lutman, M. E. (2003). The effect of speech presentation level on measurement of auditory acclimatization to amplified speech. Journal of the Acoustical Society of America, $114,484-495$.

Nabelek, A. K., Freyaldenhoven, M. C., Tampas, J. W., et al. (2006). Acceptable noise level as a predictor of hearing aid use. Journal of the American Academy of Audiology, 17, 626-639.

Nabelek, A. K., Tucker, F. M., Letowski, T. R. (1991). Toleration of background noises: relationship with patterns of hearing aid use by elderly persons. Journal of Speech and Hearing Research, $34,679-685$. 
Olsen, S. Ф., Brännström, K. L. (2014). Does the acceptable noise level (ANL) predict hearing-aid use? International Journal of Audiology, 53, 2-20.

Palmer, C. V., Bentler, R. A., Mueller, H. G. (2006). Amplification with digital noise reduction and the perception of annoying and aversive sounds. Trends in Amplification, 20, 95-104.

Palmer, C. V., Nelson, C. T., Lindley, G. A. (1998). The functionally and physiologically plastic adult auditory system. Journal of the Acoustical Society of America, 103, 17051721.

Pearsons, K. S., Bennett, R. L., Fidell, S. (1977). Speech levels in various environments (EPA-600/1-77025). Washington, DC: Environmental Protection Agency.

Pichora-Fuller, M. K., Singh, G. M. (2006). Effects of age on auditory and cognitive processing: implications for hearing aid fitting and audiologic rehabilitation. Trends in Amplification, 10, 29-59.

Rabbit, P. M. A. (2002). Aging and Cognition. In H. Pashler, D. Medin (Eds.), Steven's Handbook of Experimental Psychology: Wiley.

Robinson, D. W. (1991). Long-term repeatability of the pure-tone hearing threshold and its relation to noise exposure. British Journal of Audiology, 25, 219-235.

Rogers, D. S., Harkrider, A. W., Burchfield, S. B., et al. (2003). The influence of listener's gender on the acceptance of background noise. Journal of the American Academy of Audiology, 14, 372-382.

Saunders, G. H., Cienkowski, K. M. (1997). Acclimatization to hearing aids. Ear \& Hearing, 18, 129139.

Skagerstrand, Å., Stenfelt, S., Arlinger, S., et al. (2014). Sounds perceived as annoying by hearing-aid users in their daily soundscape. International Journal of Audiology, 53, 259-269.

Taylor, K. S. (1993). Self-perceived and audiometric evaluations of hearing aid benefit in the elderly. Ear \& Hearing, 14, 390-394.

Turner, C. W., Bentler, R. A. (1998). Does hearing aid benefit increase over time? Journal of the Acoustical Society of America, 104, 3673-3674.

Tyler, R. S., Summerfield, A. Q. (1996). Cochlear Implantation: Relationships with research on auditory deprivation and acclimatization. Ear \& Hearing, 17, 38S-50S.

van Grootel, M. W. W., Andringa, T. C., Krijnders, J. D. (2009). DARES-G1: Database of Annotated Real-world Everyday Sounds. In Proceedings of the NAG/DAGA Meeting. Rotterdam.

Wechsler, D. (2011). Wechsler Abbreviated Scale of Intelligence - Second Edition. Oxford: Pearson Assessment.

Wong, P. C. M., Jin, J. X., Gunasekera, G. M., et al. (2009). Aging and cortical mechanisms of speech perception in noise. Neuropsychologia, 47, 693-703.

Yund, E. W., Roup, C. M., Simon, H. J., et al. (2006). Acclimatization in wide dynamic range multichannel compression and linear amplification hearing aids. Journal of Rehabilitation Research \& Development, 43, 517-536. 
714 Figure 1. Mean hearing thresholds for new and experienced hearing aid users. Error

715 bars show $\pm 1 S D$ about the mean.

716

717 Figure 2. Mean real ear insertion gain (REIG; dashed line) and prescribed gain

718 targets (grey) for $65 \mathrm{~dB}$ input signal level. Error bars show $\pm 1 \mathrm{SD}$ about the mean.

719

720 Figure 3. Speech in noise performance for experienced and new hearing aid users

721 (sub sample with pure tone average $>40 \mathrm{~dB} \mathrm{HL}$ and $\mathrm{HA}$ use $>6$ hours/day). Error

722 bars show \pm 1 SD about the mean.

723

724 Figure 4. Distraction task performance (digit recall in noise minus recall in quiet).

725 Error bars show $\pm 1 S D$ about the mean.

726

727 Figure 5. Annoyance and distraction ratings of background noise during the

728 distraction task. Higher scores indicate greater annoyance/distraction. Error bars

729 show \pm 1 SD about the mean.

730

731 Figure 6. Scatter plots showing correlates of change in aided speech recognition in

732 noise for all new hearing aid users $(n=35)$

733

734

735 


\section{Left ear}

Frequency $(\mathrm{Hz})$

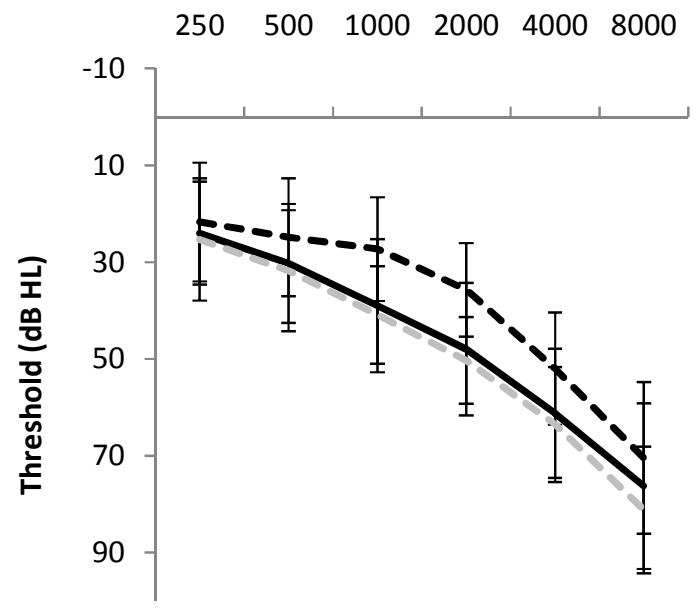

\section{Right ear}

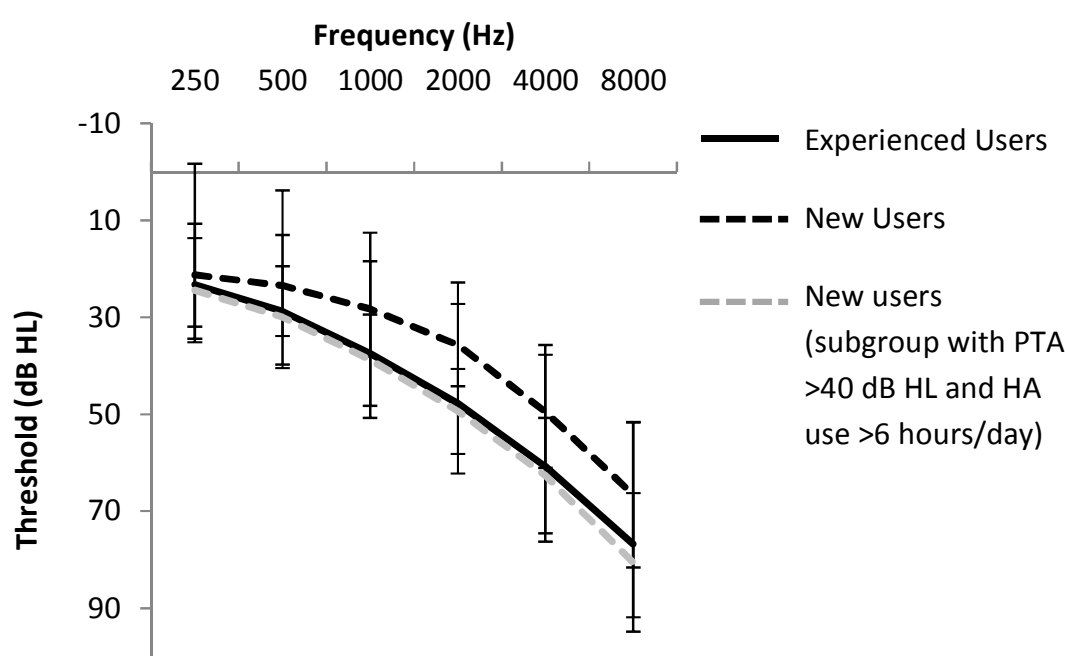




\section{Left ear}

\section{New users}

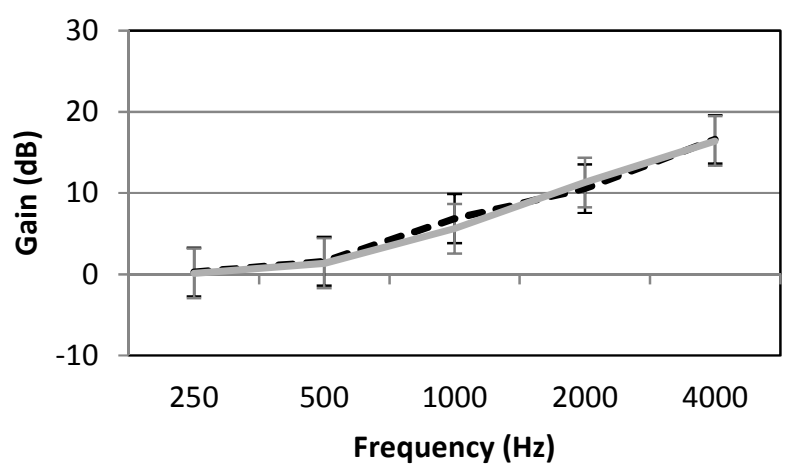

\section{Experienced users}

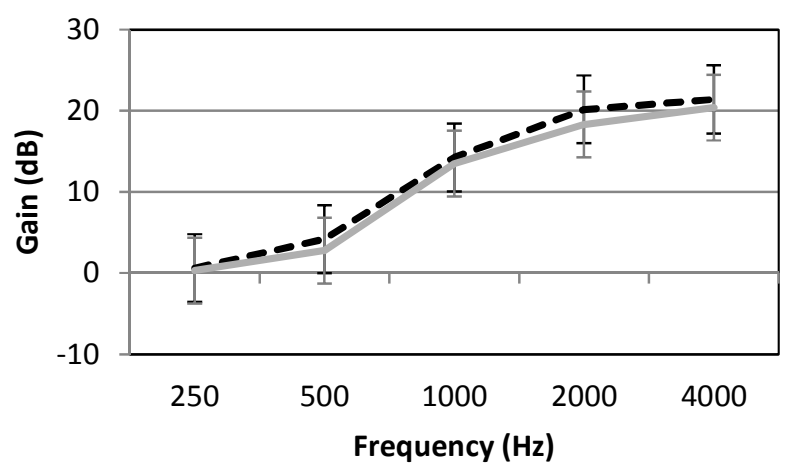

Right ear
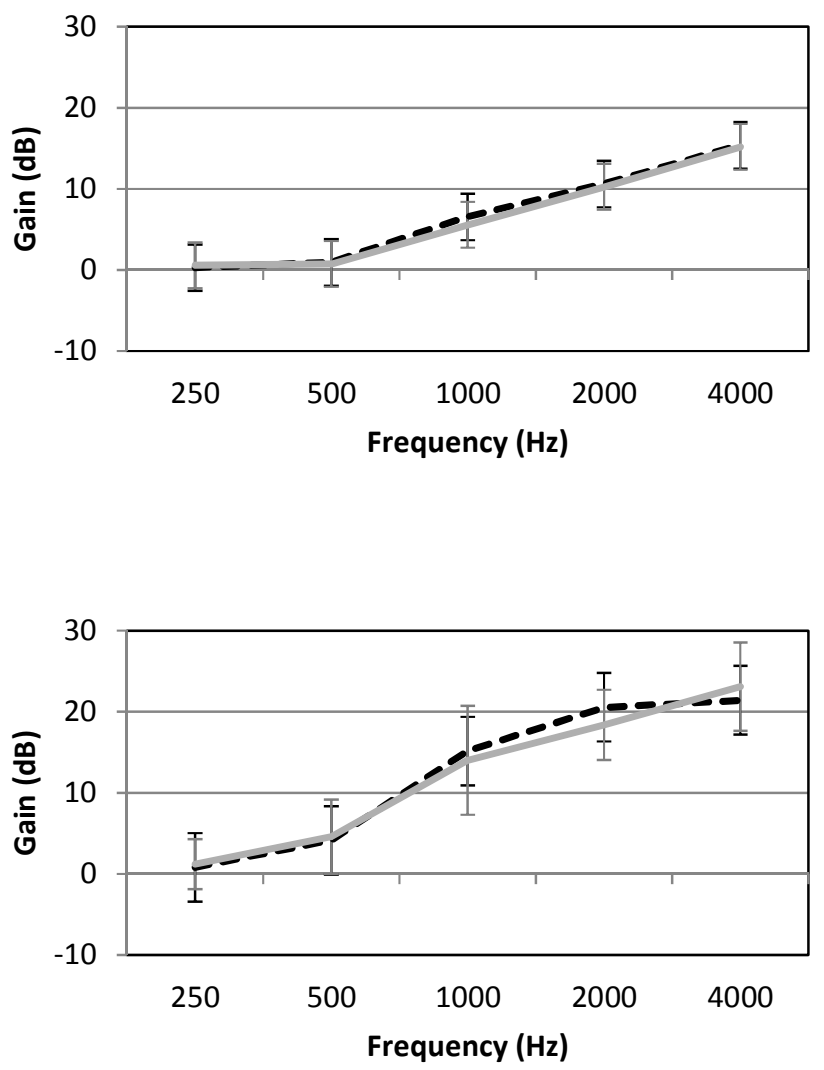

745

746

747

748

749 


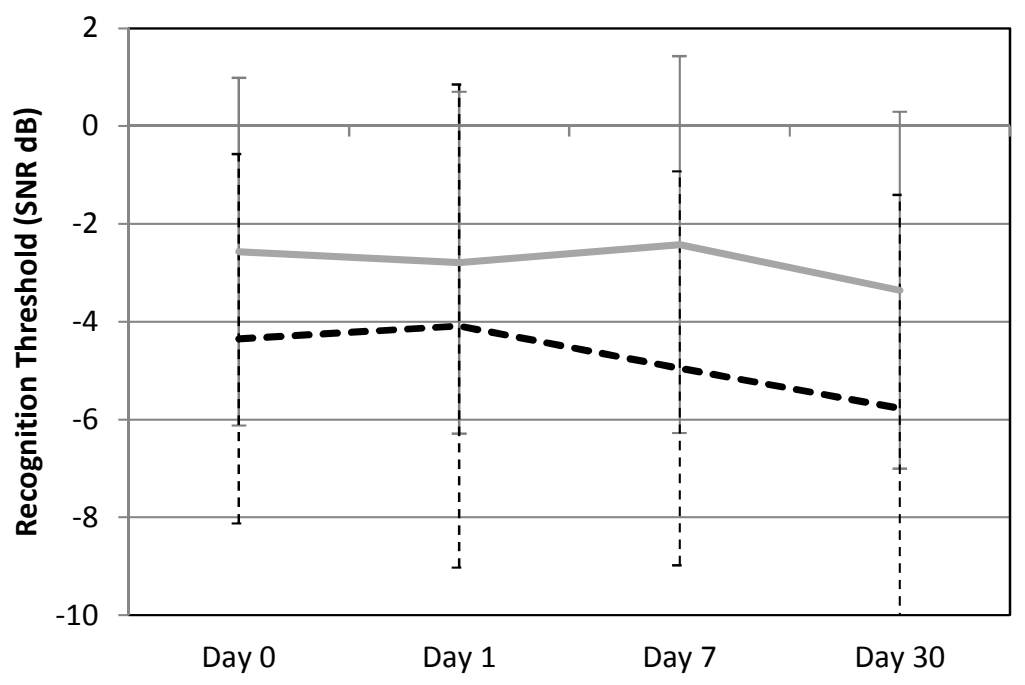

751

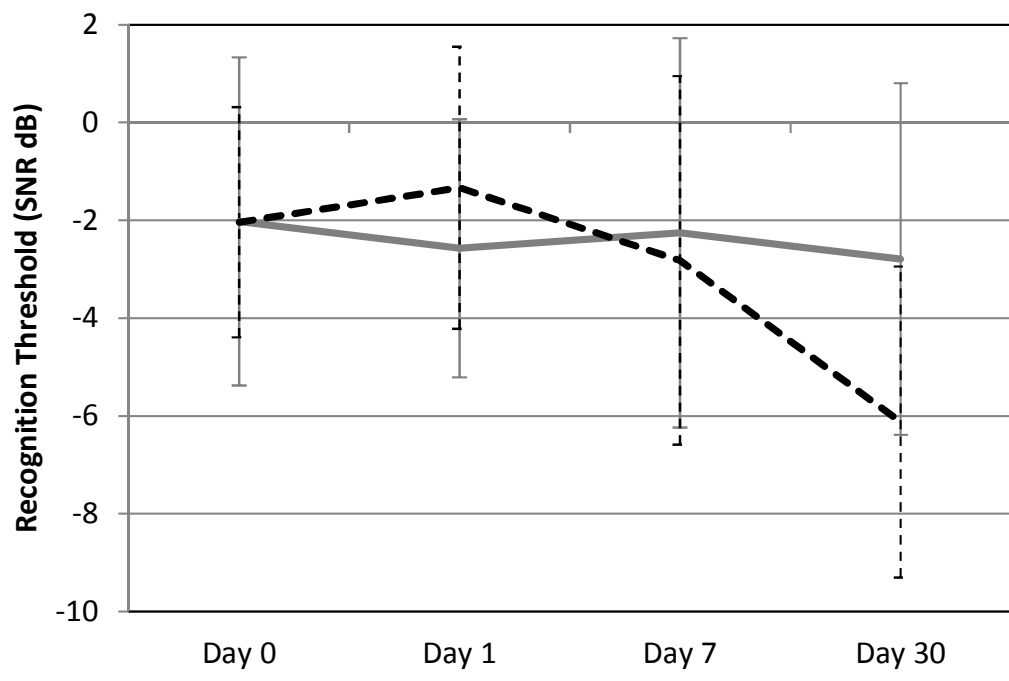

753

754

755

756 a. All participants

Experienced Users $(n=19)$

-ט-new Users $(n=34)$

b. Participants with hearing loss $>40 \mathrm{~dB} \mathrm{HL}$ and who use their hearing aids $>6$ hours per day

Experienced Users $(n=16)$

- ב- New Users $(n=10)$ 


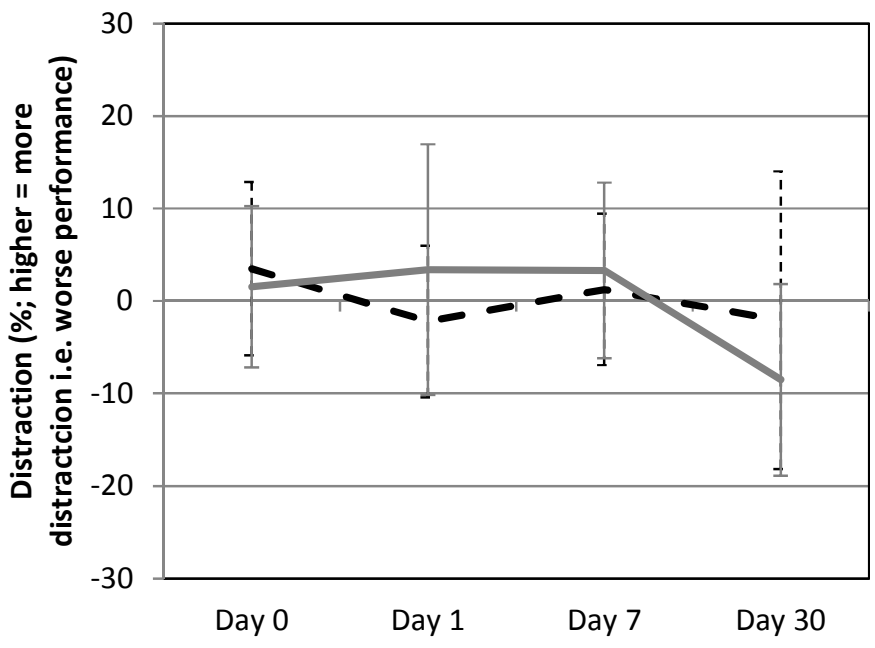

Experienced Users; $\mathrm{n}=20$

--2. New Users; $n=35$

758

759

760 
Annoyance

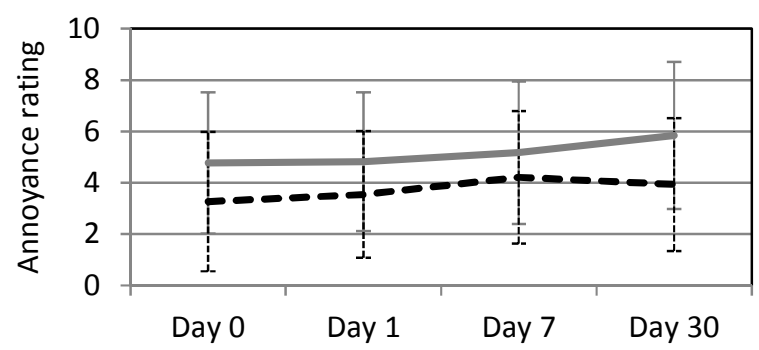

762

763

764

765

766

Experienced users; $n=20$

New users; $n=35$
Distraction

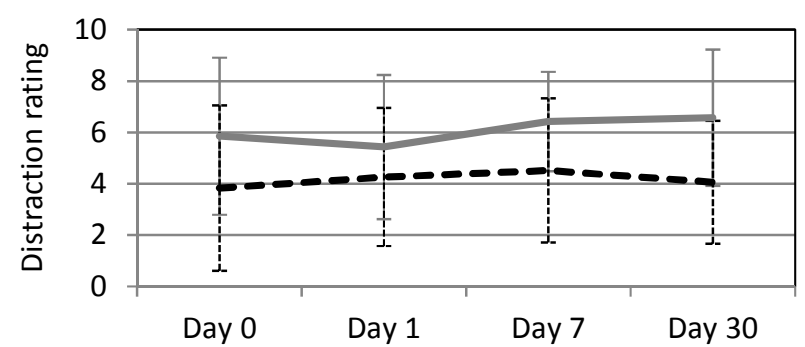



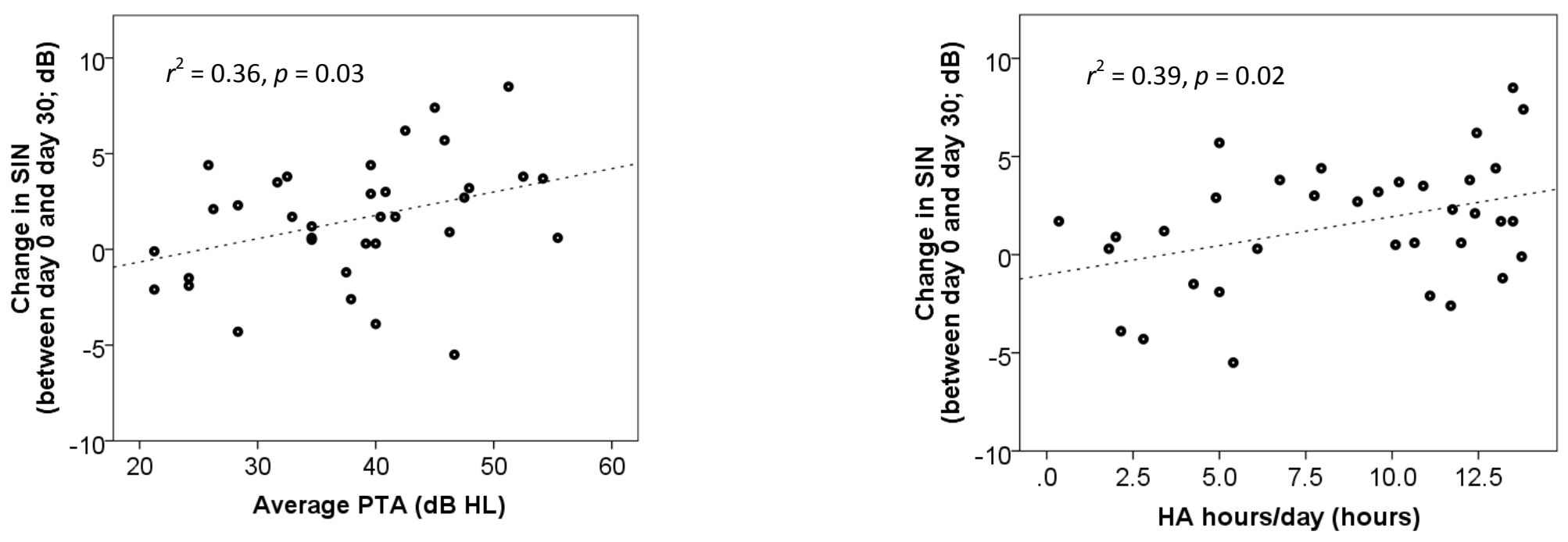

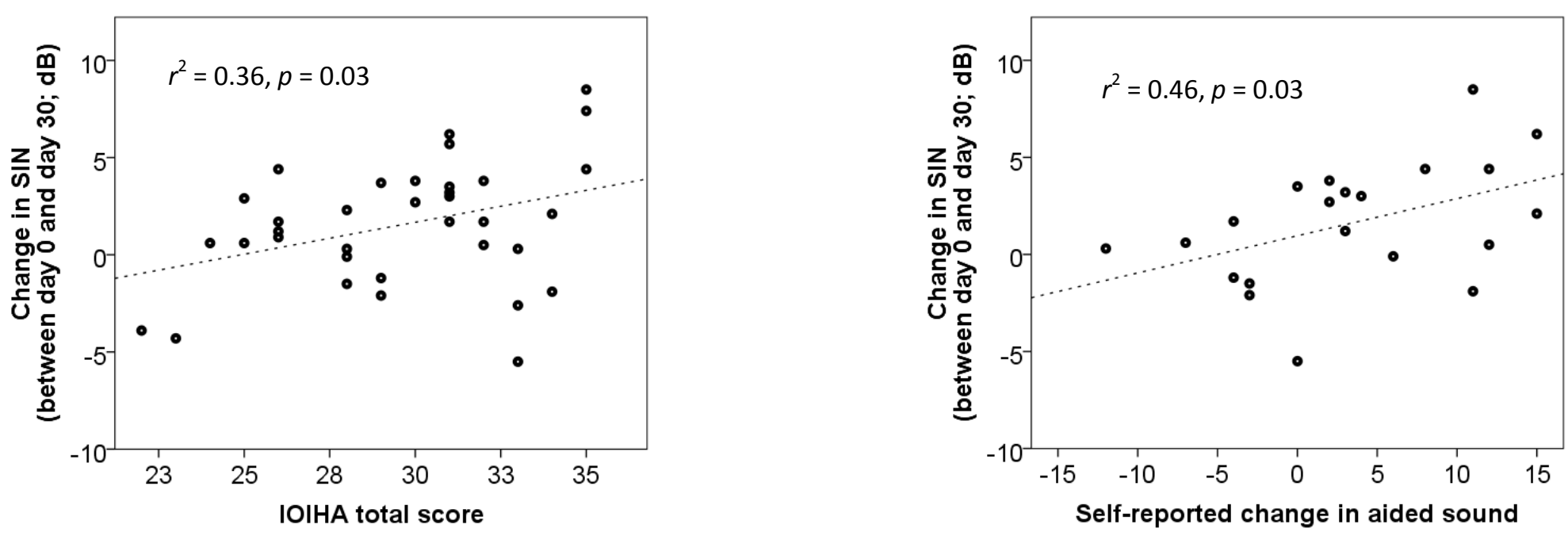
Distraction and acclimatization to hearing aids

768 